Running head: LITTLE BETWEEN-CULTURE VARIANCE IN IMPRESSIONS

\title{
Little Between-Region and Between-Country Variance When Forming Impressions of Others
}

\author{
Neil Hester ${ }^{1}$, Sally Y. Xie ${ }^{1}$, \& Eric Hehman ${ }^{1}$ \\ ${ }^{1}$ Department of Psychology, McGill University, Montreal, QC
}

In press at Psychological Science

\section{Corresponding Author:}

Neil Hester

@ neilrhester

neilrhester@gmail.com

\section{Author Contributions:}

Conceived research: NH, EH. Methodology: NH, EH. Data Curation: NH. Analysis: NH, SYX. Writing - Original Draft: NH. Writing - Review and Editing: All authors.

Revisions: All authors.

\section{Open Data:}

Our data, syntax, and supplemental materials are available at https://osf.io/gry69/.

\section{Research Support:}

This research was supported by a SSHRC Insight Development Grant (430-2016-00094) to $\mathrm{EH}$. 


\begin{abstract}
To what extent are perceivers' first impressions of others dictated by cultural background versus personal idiosyncrasies? To address this question, we analyzed a globally diverse dataset containing 11,481 adult participants' ratings of 120 targets across 45 countries $(2,597,624$ total ratings). Across ratings of 13 traits, we find that perceivers' idiosyncratic differences accounted for $\sim 29 \%$ of variance and impressions on their own and $\sim 16 \%$ in conjunction with target characteristics. However, country- and region-level differences, here a proxy for culture, accounted for on average $3.2 \%$ (i.e., both alone and in conjunction with target characteristics). We replicated this pattern of effects in a pre-registered analysis on an entirely novel dataset containing 7,007 participants' ratings of 100 targets across 41 countries (24,886 total ratings). Together, this work suggests that perceivers' impressions of others are largely dictated by their individual characteristics and local environment, rather than their cultural background.
\end{abstract}

keywords: impression formation, person perception, cross-cultural psychology, face perception, geographical analysis 
To what extent are perceivers' impressions of others dictated by cultural background versus personal idiosyncrasies? We use a first-of-its-kind globally diverse dataset to examine to what extent higher-order culture-operationalized as region and country of residencecontributes to variation in first impressions of faces. We then conduct a preregistered replication of our findings using an independent dataset.

\section{Sources of Variance in Impression Formation}

Perceivers' impressions are influenced by myriad factors: characteristics of the target, characteristics of the perceiver, and perceiver-by-target interactions (Freeman \& Ambady, 2011; Hehman et al., 2017; Kenny \& Albright, 1987; Kunda \& Thagard, 1996; Todorov et al., 2015). How target characteristics such as facial features influence impressions is the best-documented of these three sources (Hehman et al., 2019), with hundreds of studies investigating how specific facial features or other physical characteristics give rise to impressions of attractiveness, trustworthiness, dominance, and other traits (Hehman et al., 2014; Holzleitner et al., 2019; A. L. Jones \& Jaeger, 2019; Oosterhof \& Todorov, 2008; Todorov et al., 2015; Vernon et al., 2014). This body of work is framed by theories about why target appearance influences impressions, such as overgeneralization hypothesis (Zebrowitz et al., 2003) and evolutionary theories of sexual selection (Thornhill \& Gangestad, 1999).

Perceiver characteristics, though less understood, are also central to modern models of social cognition (Brewer, 1988; Bruce \& Young, 1986; Brunswik,1952; Correll, Hudson, Guillermo, \& Earls, 2016; Fiske \& Neuberg, 1990; Freeman \& Ambady, 2011; Haxby, Hoffman, \& Gobbini, 2000; Kenny \& Albright, 1987; Kunda \& Thagard, 1996; West \& Kenny, 2011). Perceiver characteristics consist of any way in which one perceiver differs from another. Differences might be trait-level, such as personality or concept knowledge, or state-level, such as 
affective state or surrounding environment. Indeed, recent work has shown that people differ in their beliefs about trait co-occurrence (i.e., "how friendly is someone who is intelligent?"), which explains considerable perceiver-level variation in first impressions (Stolier et al., 2018, 2020). Those forming impressions are not blank canvasses onto which targets project impressions; instead, observers actively interpret their world through individual lenses.

Finally, perceiver-by-target interactions describe when impressions depend on features of both the perceiver and the target. This might include differences in trait ratings from stereotypes (e.g., beliefs that Black people are athletic) as well as from idiosyncratic links between features and traits (e.g., finding red hair attractive). These perceiver-by-target interactions are central to intergroup research, in which individuals in different groups differentially evaluate stimuli that vary by race, gender, or other identities. For example, perceivers higher in ambivalent sexism perceive men in egalitarian (rather than stereotypic) relationships as less warm and competent (McCarty \& Kelly, 2015). Dynamic models of impression formation map out how target and perceiver characteristics continually interact across multiple levels of processing during impression formation (Freeman et al., 2020; Kunda \& Thagard, 1996).

\section{Differences in the Importance of Variance Sources}

Despite the historical focus on target-level variance, recent work has found that perceiver and perceiver-by-target characteristics each play a larger role in overall first impressions (20$25 \%$ of the variance) than target characteristics (10-15\% of the variance; Hehman et al., 2017; Hönekopp, 2006; Xie et al., 2019). In this work, cross-classified multilevel models were used to decompose impressions into variance attributable to the target, perceiver, and perceiver-by-target interactions (Kenny et al., 2006; Raudenbush \& Bryk, 2002). Understanding the relative contribution of different sources of impression variance is critical to impression formation 
theory. As a parallel, epidemiologists cannot effectively understand the dangers of a virus without knowing how much genetics and experience (i.e., nature vs. nurture), as well as their interaction, uniquely contribute to individual susceptibility. Similarly, to understand the extent to which perceiver- and target-level factors influence our impressions is to better understand the processes by which perceivers form impressions (see Hehman et al., 2017).

Characteristics of the perceiver, both in the form of perceiver variance and perceiver-bytarget interactions, thus account for considerable variance in any given impression. Previous research has quantified the extent of their contribution. However, it is unclear exactly what perceiver characteristics are important for guiding impression formation, because "how one perceiver differs from another" is so broad. Is the locus of this perceiver variability in the individual? Differences in how perceivers evaluate the same target may arise from idiosyncratic factors such as personal interests, experiences, and beliefs. This is at least partly the case, as demonstrated by perceiver-level variability in ratings constrained to a single country (Hehman et al., 2017).

Alternatively, the locus could be the broader context in which individuals are embedded, such as cultural beliefs that cluster as a function of one's country or global region. Because different perceivers are in different cultures when forming impressions, previous research would have identified effects of the broader environment as "perceiver-level" when in reality the true source of variance is not "in" the perceiver, but instead the broader culture and location. These higher-order clusters could potentially account for a large proportion of what has previously been identified as perceiver-level variance.

Cross-cultural research has shown important higher-order differences in first impressions (e.g., Birkás et al., 2014). Furthermore, work has argued that both the factor structure of 
trustworthiness and dominance in face perception (Jones, DeBruine, Flake ... Chartier, \& Coles, 2021) varies considerably as a function of culture, suggesting that region- and country-level cultural differences might play a large role in shaping impressions. However, other work comparing two specific cultures has found limited cultural variation in both the factor structure (Sutherland et al., 2018) and cultural variability (Zebrowitz et al., 2012) of first impressions. The present work contributes to the ongoing discussion regarding culture and first impressions by measuring cultural variability using a large and geographically diverse data set.

\section{The Present Research}

Across a broad array of domains_-social cognition, social perception, person perception, and others-researchers use trait impressions to understand how humans perceive others. Although it is important to disentangle idiosyncratic perceiver factors from systematic cultural factors in impression formation, doing so requires numerous perceivers within numerous cultures, making it difficult. We capitalize on a unique dataset of 2,597,624 trait ratings from 45 countries (Jones et al., 2021) to compare the role of between-target, between-perceiver, and between-culture differences (operationalized as country and region) in first impressions. We solidify this contribution by conducting a preregistered replication of our findings using a second dataset provided by one of the reviewers of the manuscript (Zickfeld, van de Ven, Pich, Schubert, Berkessel, Pizarro Carrasco ... Orjiakor, 2020).

\section{Study 1}

\section{Method}

\section{Open Data and Syntax}

The original data and documentation from Psychological Science Accelerator 001 (PSA001; Jones et al., 2021; Moshontz et al., 2018) are available at https://osf.io/87rbg/. Our code and 
supplemental files are available at https://osf.io/gry69/. Here, we disclose that two of the three authors of this manuscript also participated in data collection for PSA-001.

\section{Participants}

We analyzed the dataset generated by the PSA-001, a collaboration between 117 labs around the world to test the universality of the proposed basic dimensions of face perception (Oosterhof \& Todorov, 2008). We followed their preregistered data cleaning procedure which retained 11,481 participants $(69.6 \%$ women, $29.7 \%$ men, $0.7 \%$ other; mean age $=22.6$ years $)$, nested within 45 countries, nested within 11 regions (see Table 1 for list of countries and regions as defined by PSA-001; and Figure 1 for participant breakdown by country). Thus, we operationalized cultural variability as between-country and between-region variability. For more details on the selection of countries and the division into regions, see PSA-001.

Table 1

Countries by region, as reported in PSA-001.

WORLD REGION

$\operatorname{AFRICA}(\mathrm{N}=520)$

EAST AND SOUTHEAST ASIA $(\mathrm{N}=780)$

AUSTRALIA AND NEW ZEALAND ( $\mathrm{N}=1044)$

CENTRAL AMERICA AND MEXICO ( $\mathrm{N}=338)$

EASTERN EUROPE $(\mathrm{N}=809)$

MIDDLE EAST $(\mathrm{N}=503)$

USA AND CANADA $(\mathrm{N}=3312)$

SCANDINAVIA ( $N=653)$

SOUTH AMERICA $(\mathrm{N}=1388)$

UNITED KINGDOM ( $\mathrm{N}=361)$

WESTERN EUROPE $(\mathrm{N}=1862)$

\section{COUNTRIES WITHIN REGION}

Kenya, Nigeria, South Africa

China, India, Malaysia, Taiwan, Thailand

Australia, New Zealand

El Salvador, Mexico

Hungary, Lithuania, Poland, Russia, Serbia,

Slovakia

Iran, Israel, Turkey

Canada, USA

Denmark, Finland, Norway, Sweden

Argentina, Brazil, Chile, Colombia, Ecuador

England, Scotland, Wales

Austria, Belgium, France, Germany, Greece,

Italy, the Netherlands, Portugal, Spain,

Switzerland 
Figure 1

Distribution of participants across the 45 countries included in Study 1.
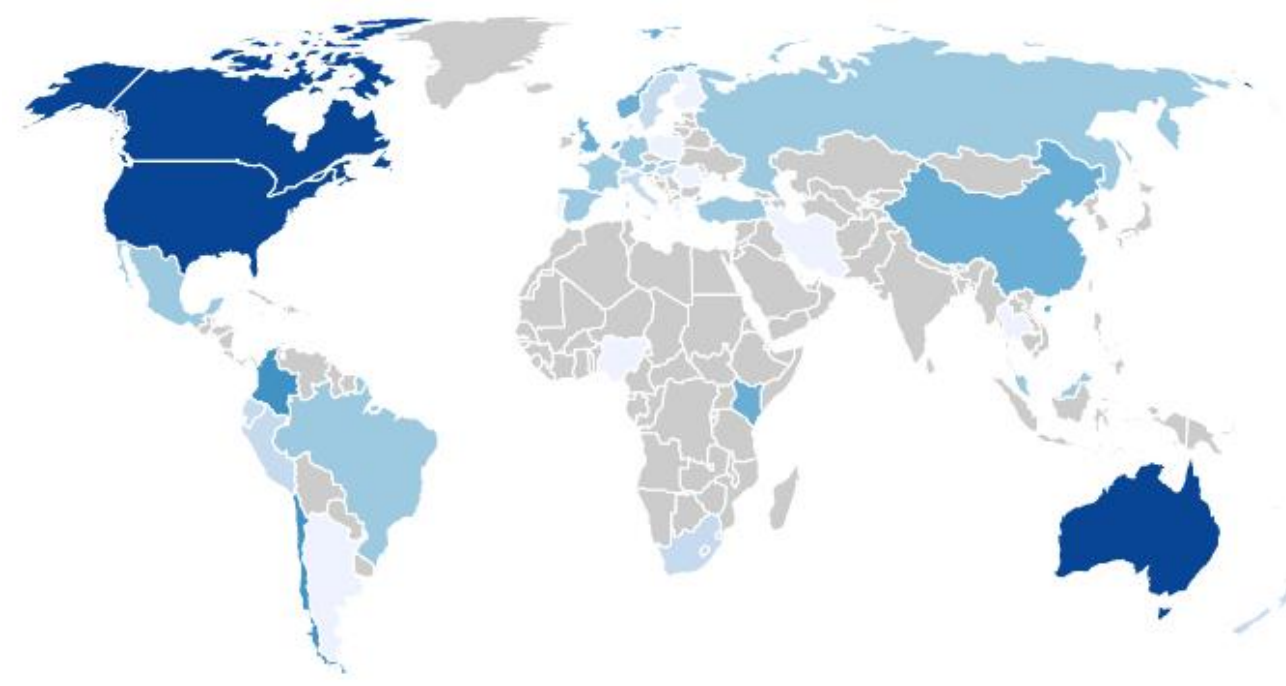

Participant Count

One challenge of global data collection was accommodating various languages. To mitigate linguistic differences as responsible for variation, twelve of the thirteen adjectives collected were accompanied by dictionary definitions (dominant was not, instead being defined as "strong, important"; Jones et al., 2021). To consider language as an alternate proxy for culture, we provide secondary analyses clustering by language rather than country and region.

\section{Procedure}

Each participant was randomly assigned to rate faces on one of thirteen traits commonly used in person perception research: aggressive, attractive, caring, confident, dominant, emotionally stable, intelligent, mean, responsible, sociable, trustworthy, unhappy, or weird. Participants completed 240 trials in which they rated neutrally-posed faces on a 7-point scale (from "Not at all" to "Very") for the assigned trait. The 240 trials were divided into two 120-trial blocks, such that participants rated each face twice, enabling the partitioning of variance of the perceiver-by-target interactions from the residual (Hehman et al., 2017). The 120 faces were 
drawn from the Chicago Face Database (Ma et al., 2015) and evenly divided across ethnicity (Asian, Black, Latine, White) and gender (female, male).

\section{Analytic Approach}

We used multilevel models to calculate the amount of variance in trait ratings attributable to specific levels of clustering (e.g., perceiver, target, country, region). In these null or interceptonly models, participants' ratings of stimuli on the dimension of interest (e.g., trustworthiness) served as the single dependent variable. The structure and size of the PSA-001 data allowed us to estimate four-level models for each trait: 2,597,624 trait ratings (Level 1) were cross-classified by 11,481 perceivers and 120 targets (Level 2). Perceivers were nested within 45 countries (Level 3) and countries within 11 regions (Level 4). Models were estimated using R packages lme4 (Bates et al., 2015) and estimates from models that did not converge were confirmed in brms (Bürkner, 2017).

This model can be defined with Equation 1 using four levels:

$$
\begin{aligned}
& \text { Level 1: } Y_{i(j k) l m}=\pi_{0(j k) l m}+e_{i(j k) l m} \\
& \text { Level 2: } \pi_{0(j k) l m}=\beta_{000 l m}+r_{0 j 000}+r_{00 k 00}+d_{0(j k) 00}+d_{00 k l 0}+d_{00 k 0 m} \\
& \text { Level 3: } \beta_{000 l m}=\gamma_{0000 m}+r_{000 l 0} \\
& \text { Level 4: } \gamma_{0000 m}=\theta_{00000}+u_{0000 m}
\end{aligned}
$$

At Level $1, Y_{i(j k) l m}$ is our dependent variable of interest: a rating on dimension $i$ (e.g., trustworthiness) by perceiver $j$ of target $k$, in which perceivers are nested within $l$ countries within $m$ regions. The intercept, $\pi_{0(j k) l m}$, is the expected value of this rating, and the error term, $e_{i j k}$, has its own associated variance, $\sigma^{2}$. At Level 2 of the model, the intercept $\pi_{0(j k) l m}$ is modeled as an outcome that varies across perceivers and targets, which allows the total variance of the model to be partitioned into that attributable to perceivers and targets. The group mean of perceiver ratings, $\beta_{000 \mathrm{~lm}}$, represents the expected value of the rating made by perceivers in 
country $l$ (nested within region $m$ ) across all targets. The residual, $r_{0 j 000}$, is the deviation of perceiver $j$ from the mean score of their respective country (averaged across all targets), which has variance $\tau_{j 00}$. The other residual, $r_{00 k 00}$, is the residual of target $k$, or the difference between the grand mean and the rating of target $k$ averaged across all perceivers; these residuals have variance $\tau_{k 00}$. The random effect, $d_{0(j k) 00}$ represents the interaction between perceiver and target variance in the model and can be partitioned from error when a perceiver rates the same target at least twice (i.e., repeated measures within a perceiver and target). The other two random effects are $d_{00 k l 0}$, representing the interaction between target and country-level variance in ratings, and $d_{00 \mathrm{k} 0 \mathrm{~m}}$, representing the interaction between target and region-level variance in ratings.

At Level 3 of the model, the expected value for the group mean, $\beta_{000 l m}$, is a function of the regional mean score, $\gamma_{0000 m}$ (i.e., the average rating across countries within each region), plus each country's residual from the mean rating of their region, $r_{000 l 0}$, which has variance $\tau_{l 00}$. Finally, at Level 4 , the expected value for the regional mean, $\gamma_{0000 m}$, is a function of the grand mean across all clusters (i.e., average rating across all targets and perceivers across all countries and regions), plus each region's residual from that grand mean, $u_{0000 m}$, with variance $\tau_{m 00}$.

Thus, we are estimating 8 variance terms in the model: variance across perceivers, $\tau_{j 00}$, variance across targets, $\tau_{k 00}$, variance across countries, $\tau_{l 00}$, variance across regions, $\tau_{m 00}$, variance of the interaction between perceivers and targets, $\tau_{b 00}$, variance of the interaction between targets and countries, $\tau_{c 00}$, variance of the interaction between targets and regions, $\tau_{d 00}$, and the level-1 error term, $\sigma^{2}$. Together, these terms comprise $100 \%$ of the variance in ratings on any dimension. 
By looking at the size of each variance component relative to the total variance, we can calculate the proportions of variance that come from different elements of the model in an intraclass correlation coefficient (ICC; McGraw \& Wong, 1996; Shrout \& Fleiss, 1979). For example, target-ICC is calculated as the proportion of variance attributable to perceiver characteristics (Equation 2):

$$
\mathrm{ICC}_{\text {target }}=\frac{\tau_{k 00}}{\tau_{k 00}+\tau_{j 00}+\tau_{l 00}+\tau_{m 00}+\tau_{b 00}+\tau_{c 00}+\tau_{d 00}+\sigma^{2}}
$$

This approach descends from the social relations model in dyadic impressions (Kenny et al., 2006). Using this approach, we can determine how much variance is attributable to individual factors (that do not correspond to location) versus cultural factors (that do correspond to location).

Consider an example in which country-ICC was .80. This result would indicate that $80 \%$ of the variance in a particular trait impression is due to between-country differences, suggesting that people in different countries were mostly drawing on shared cultural experiences when forming impressions. In contrast, if country-ICCs were very low (e.g., .02), only $2 \%$ of the variance in trait impressions would be due to between-country differences, suggesting that other sources of variance were primarily driving the impressions. This latter situation highlights the importance of knowing the ICC. If an ICC is $2 \%$, no matter how many country-level variables are included in a model, they can together explain at most $2 \%$ of the variance in the trait impression. Such a situation would reveal that between-culture differences should perhaps not be a major focus of future research concerned with predicting and explaining people's first impressions. Importantly, ICCs do not identify which variables are related to dependent variables, but only quantify to what extent variance comes from different levels, and therefore how to develop future theoretical models to best explain that variance. 
Study 1 analyses were not preregistered. Instead, we tested an identical model across 13 different traits, providing multiple conceptual replications. We estimated ICCs for perceivers, targets, and perceiver-by-target combinations. Uniquely, we also estimated ICCs for countries, regions, country-by-target combinations, and region-by-target combinations. 95\% confidence intervals were bootstrapped around each ICC (Xie et al., 2019). Together, these ICCs allowed us to quantify how much variance in trait ratings is attributable to between-country or betweenregion differences, and to what extent a rating depended simultaneously on characteristics of the target and country/region.

\section{Results}

Figure 2 displays the ICCs for all thirteen traits. Although ICCs vary minorly across trait (see Supplementary File for full reporting), they follow a consistent pattern. Across traits, perceiver differences accounted for largest amount of variance $\left(M_{\mathrm{ICC}}=29.1 \%\right.$; Range $_{I C C}=21$ $35 \%)$, followed by perceiver-by-target differences $\left(M_{\mathrm{ICC}}=15.6 \% ;\right.$ Range $\left._{I C C}=15-20 \%\right)$ and target differences $\left(M_{\mathrm{ICC}}=10.0 \% ;\right.$ Range $\left._{I C C}=8-15 \%\right)$. Country and region differences, whether alone or interacting with target, accounted for little variance in trait ratings (country $M_{\mathrm{ICC}}=$ $1.4 \%$, Range $e_{I C C}=0-4 \% ;$ region $M_{\mathrm{ICC}}=0.7 \% ;$ Range $e_{I C C}=0-2 \% ;$ country-by-target $M_{\mathrm{ICC}}=0.6 \%$, Range $_{I C C}=0-1 \% ;$ region-by-target $M_{\mathrm{ICC}}=0.5 \%$, Range $\left._{I C C}=0-1 \%\right)$. 
Figure 2

ICCs by cluster type and trait, Study 1.
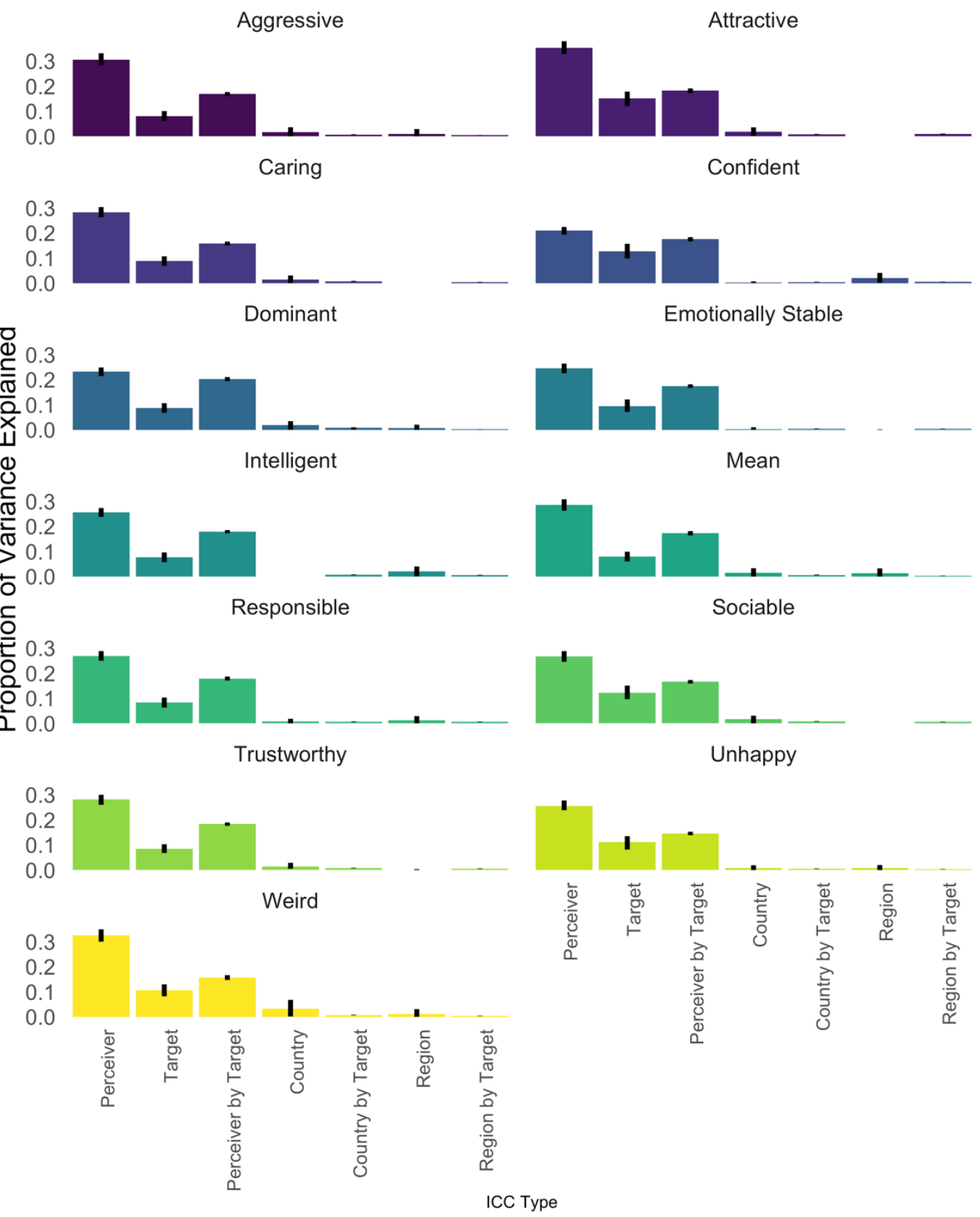

Note. Error bars represent $95 \%$ confidence intervals. Colors vary by trait and are provided for visual clarity. 
The faces that participants had rated varied by ethnicity (i.e., Asian, Black, Latine, White) and sex (i.e., female, male). One possibility was that while between-culture and betweenregion differences didn't matter in the aggregate, perhaps their effect would be more substantial on specific ethnic and gender subgroups given cultural variation in ethnic and gender stereotypes. To test this possibility, in supplementary analyses we fit identical models for each subgroup (i.e., Asian Female, Asian Male, Black Female, Black Male, Latine Female, Latine Male, White Female, White Male). Yet between-culture and between-region differences were consistently not important for impressions of any subgroup. Full reporting of results is available in the Supplementary Materials.

We additionally fit models in which we replaced country (Level 3) and region (Level 4) with language (Level 3) to test whether language as a clustering variable yielded higher ICCs. It did not, yielding similarly low ICCs to country and region. These analyses are documented in the Supplementary Materials.

Together, results indicate that differences between country or region, which we used to operationalize high-level cultural differences, do not account for variance in trait ratings.

\section{Study 2}

\section{Method}

\section{Preregistration, Open Data, and Syntax}

Study 2 is a preregistered conceptual replication test of Study 1 using novel data from an investigation of the interpersonal effects of emotional crying across 41 countries (Zickfeld et al., 2020). The preregistration is available at https://osf.io/g59u6. The key hypothesis in our preregistration is that region, region-by-target, country, and country-by-target clusters would 
account for no more than $8 \%$ of the total variance in ratings across all eight traits in the replication dataset.

The most recent data and documentation from Zickfeld and colleagues' investigation (2020) are available at https://osf.io/fj9bd/. Our code and supplemental files are available at https://osf.io/gry69/.

\section{Participants}

For our preregistered replication, we analyzed a dataset from an investigation of the interpersonal effects of emotional crying across 41 countries (Zickfeld et al., 2020). We followed their preregistered data cleaning procedure which retained 24,886 trait ratings of 7,007 participants ( $68.9 \%$ women, $30.7 \%$ men, $0.7 \%$ other; mean age $=28.2$ years $)$, nested within 41 countries, nested within 11 regions (see Table 2 for list of countries and regions). Translation of traits followed the recommendations from PSA-001 (Jones et al., 2021).

Table 2

Countries by region in Study 2, categorized using the methodology of PSA-001.

WORLD REGION

AFRICA ( $=352)$

EAST AND SOUTHEAST ASIA ( $N=1192)$

AUSTRALIA AND NEW ZEALAND ( $\mathrm{N}=156)$

CENTRAL AMERICA AND MEXICO ( $\mathrm{N}=298)$

EASTERN EUROPE $(\mathrm{N}=608)$

MIDDLE EAST $(\mathrm{N}=1141)$

USA AND CANADA $(\mathrm{N}=302)$

SCANDINAVIA ( $N=459)$

SOUTH AMERICA $(\mathrm{N}=488)$

UNITED KINGDOM ( $\mathrm{N}=159)$

WESTERN EUROPE $(N=1852)$

\section{COUNTRIES WITHIN REGION}

Nigeria, South Africa

China, India, Japan, Malaysia, Philippines,

Singapore, South Korea, Thailand

Australia, New Zealand

Colombia, Mexico

Bosnia \& Herzegovina, Croatia, Hungary,

Poland, Serbia, Slovakia,

Israel, Pakistan, Turkey, United Arab Emirates

Canada, USA

Finland, Norway

Argentina, Brazil, Chile, Peru

Ireland, UK

Austria, France, Germany, Greece, the

Netherlands, Portugal, Spain 


\section{Procedure}

Participants completed 4 trials in which they rated neutrally-posed faces from the Chicago Face Database (White, Black, Latine, East Asian; Ma et al., 2015) and the Bogazici Face Database (Turkish; Saribay et al., 2018). Half of these faces were digitally edited to add tears (the facial expressions themselves were not edited) to address key hypotheses that motivated the original collection of the data. Furthermore, the study included manipulations of both situational valence and social context. For full details, please refer to Zickfeld and colleagues (2020). Although these manipulations might introduce additional variability on both the perceiver and target levels, they do not preclude estimations of variance at the country and region levels, which are the key estimates for our preregistered analyses.

For each face, participants provided ratings on a 7-point scale for the following adjectives: attractive, capable, competent, dominant, friendly, honest, reliable, and warm. Participants also provided other ratings unrelated to our hypotheses, which we have not included.

\section{Analytic Strategy}

We used the same analytic strategy as in Study 1, with the exclusion of the perceiver-bytarget estimate of variance (to dissociate this term from the error term, two perceiver ratings of each target are necessary).

\section{Results}

Figure 3 displays the ICCs for all eight traits. Although ICCs vary minorly across trait (see Supplementary File for full reporting), they follow a consistent pattern. Across traits, perceiver differences accounted for largest amount of variance $\left(M_{\mathrm{ICC}}=28.9 \%\right.$; Range $_{I C C}=21$ $35 \%)$. Target differences accounted for less variance than in the PSA-001 study $\left(M_{\mathrm{ICC}}=3.1 \%\right.$; Range $_{I C C}=1-9 \%$ ); this difference might be due to the low number of target stimuli per 
participant. Country and region differences, whether alone or interacting with target, accounted for little variance in trait ratings (country $M_{\mathrm{ICC}}=2.7 \%$, Range $e_{I C C}=2-4 \%$; region $M_{\mathrm{ICC}}=1.0 \%$; Range $_{I C C}=0-2 \% ;$ country-by-target $M_{\mathrm{ICC}}=0.3 \%$, Range $e_{I C C}=0-1 \% ;$ region-by-target $M_{\mathrm{ICC}}=$ $0.4 \%$, Range ICC $=0-1 \%)$. We found evidence consistent within our preregistered hypothesis that country, region, country-by-target, and region-by-target clusters would account for less than $8 \%$ of the variance in face ratings $\left(M_{I C C}=4.4 \%\right.$, Range $\left._{I C C}=3-6 \%\right)$. In general, results were in a pattern very similar to Study 1. 
Figure 3

ICCs by cluster type and trait, Study 2.

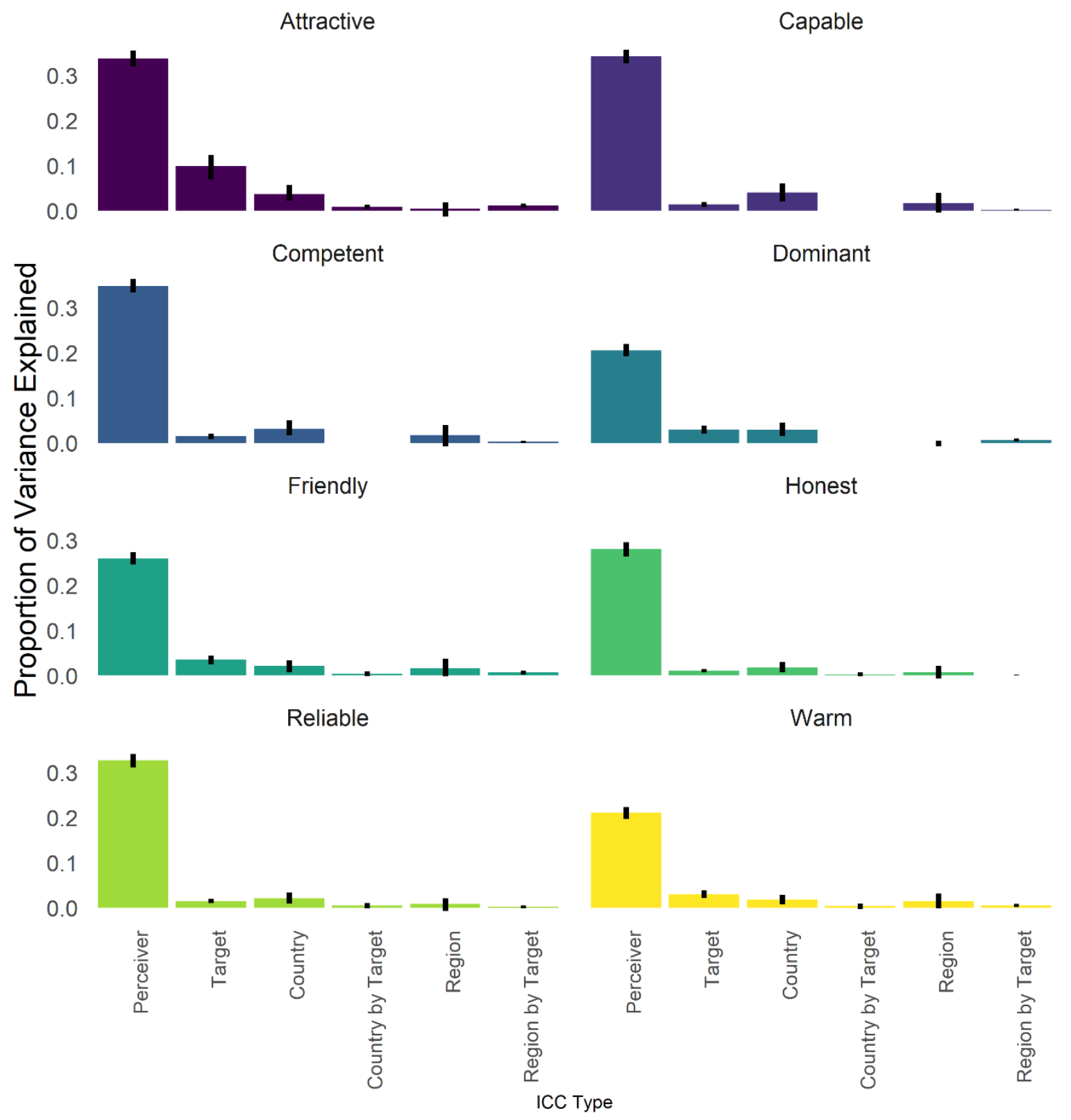

Note. Error bars represent $95 \%$ confidence intervals. Colors vary by trait and are provided for visual clarity. 


\section{General Discussion}

Analysis of over 2,500,000 trait ratings suggests that between-culture differences account for minimal variance in trait impressions inferred from faces. Consistent with previous work (Hehman et al., 2017; Hönekopp, 2006; Xie et al., 2019) we find that perceiver and perceiver-bytarget characteristics are larger sources of variance in impressions than what targets "look like." Culture, operationalized as region and country, does not play a substantial role in the outcome of impression formation, accounting for at most $5 \%$ of the variance in any given trait in our sample (which, though the most diverse to date, still did not substantively sample from Africa, Asia, and from older adult populations). Researchers wishing to examine between-culture variation in impressions might keep this upper threshold in mind.

The present results converge with recent research highlighting individual-centered variance in how impressions are formed. For example, research adopting a twin-study design partitioned the variability of personal environment and genetics in forming impressions of trustworthiness, attractiveness, and dominance. Results indicated that genetics explained little variability relative to one's personal environment (Sutherland et al., 2020), which encompasses local factors related to one's upbringing and family/community environment — and are likely to drive the observed perceiver-level differences. Other work suggests that individuals' conceptual trait spaces (i.e., the ways that different traits correlate with each other) are learned from actual personality structure in one's environment, which may explain the similar structure observed in face, person knowledge, and stereotype domains (Stolier et al., 2020). This work, together with the present results, supports the importance of individual variability in shaping the outcome of impression formation, relative to genetic and cultural variability. 


\section{Cultural Heterogeneity in Factor Structure versus Partitioned Variance}

The research generating this data found regional heterogeneity in the factor structure underlying impression formation (Jones et al., 2021). It is important to clarify that the present results are not at odds with this conclusion. Whereas we find that between-culture differences account minimally for variance in an impression of any single trait, work examining factor structure focuses on how different trait impressions covary. Identifying the source of variance in perceivers' impressions is distinct from questions about structure. Although structure appears to vary regionally (Jones et al., 2021; Wang et al., 2019), variance in any individual's trait ratings mostly arises from idiosyncratic perceiver and target differences.

This contrast implies that cross-cultural research — and any work that explores group differences - should treat questions about factor structure and questions about partitioned variance as theoretically distinct. One broad possibility is that the latent factor structure of impressions tends to vary by higher-order factors such as culture, but that the variance in these impressions tends to vary by lower-order perceiver and target differences. In other words, it is possible that people's concept knowledge of broad latent factors (i.e., what latent factors exist and what manifest variables reflect this latent factor) is more culturally determined, but the way that they infer a given trait from a stimulus is more individually determined — or, at least, is determined by a lower-order geography or culture (e.g., within-country regional units). Better understanding this distinction is integral for forming domain-general theories of social perception that simultaneously discuss both factor structure and individual variance (Freeman et al., 2020). 


\section{Limitations}

Our conclusions rely on several assumptions. One is that participants in each country are representative of the way in which impressions are formed in that country. For example, the preponderance of undergraduate participants may make the dataset non-representative to such a degree that we failed to capture large amounts of cultural variation. It is likely that this feature of the data produces a conservative estimate of the effect of culture. However, it seems unlikely that young participants are "unembedded" in their countries' or regions' cultures to such a degree that they wholly obscure cultural effects. Further, at least in Western samples, variance estimates from undergraduates match that of the broader population (Hehman et al., 2017).

We also assume that effects generalize to impression formation broadly, rather than only this commonly-used subset of thirteen trait impressions (Oosterhof \& Todorov, 2008). The stimuli also do not exhaustively represent the diverse populations by whom they were rated and demonstrate only "neutral" expressions. Future research might examine whether our results hold for different, more dynamic, and less controlled stimuli. Furthermore, we recognize that the samples in both studies draw from African and Asian countries in limited ways, as shown in Figure 1. The omission of these regions constrains our claims that these results generalize globally.

Finally, the present research operationalizes "culture" as between-country and betweenregion variation. Culture can vary dramatically by smaller intra-country units, and any intracountry cultural variation would be missed by the present models. Intra-cultural variation certainly exists for some individual differences. For example, U.S. states vary on Big Five personality traits (Rentfrow et al., 2008). Furthermore, because people travel and relocate, region and country as operationalizations of culture will include some measurement error that might 
lead to underestimation of country- and region-level effects. Finally, culture defined in other ways might still meaningfully impact impression formation, such as the rural-urban, liberalconservative, or poor-rich spectra. Future research could test if culture defined in these ways reveals meaningful variation not captured here.

\section{Conclusion}

For any one impression that a perceiver forms of a static face, higher-order cultural factors (that is, those organized by country or region) play a relatively small role in what this impression will be, relative to personal idiosyncrasies or low-order cultural factors. The present results suggest that the most universal aspect of first impressions is their variability across individual perceivers and targets, regardless of location or culture. 


\section{References}

Bates, D., Mächler, M., Bolker, B., \& Walker, S. (2015). Fitting linear mixed-effects models using lme4. Journal of Statistical Software, 67(1), 1-48. https://doi.org/10.18637/jss.v067.i01

Birkás, B., Dzhelyova, M., Lábadi, B., Bereczkei, T., \& Perrett, D. I. (2014). Cross-cultural perception of trustworthiness: The effect of ethnicity features on evaluation of faces' observed trustworthiness across four samples. Personality and Individual Differences, 69, 56-61. https://doi.org/10.1016/j.paid.2014.05.012

Bürkner, P.-C. (2017). brms: An R package for Bayesian multilevel models using Stan. Journal of Statistical Software, 80(1), 1-28. https://doi.org/10.18637/jss.v080.i01

Freeman, J. B., \& Ambady, N. (2011). A dynamic interactive theory of person construal. Psychological Review, 118(2), 247-279. https://doi.org/10.1037/a0022327

Freeman, J. B., Stolier, R. M., \& Brooks, J. A. (2020). Dynamic interactive theory as a domaingeneral account of social perception. In Advances in Experimental Social Psychology (Vol. 61, pp. 237-287). Elsevier. https://doi.org/10.1016/bs.aesp.2019.09.005

Hehman, E., Leitner, J. B., \& Freeman, J. B. (2014). The face-time continuum: Lifespan changes in facial width-to-height ratio impact aging-associated perceptions. Personality and Social Psychology Bulletin, 40(12), 1624-1636. https://doi.org/10.1177/0146167214552791

Hehman, E., Stolier, R. M., Freeman, J. B., Flake, J. K., \& Xie, S. Y. (2019). Toward a comprehensive model of face impressions: What we know, what we do not, and paths forward. Social and Personality Psychology Compass, 13(2), e12431. https://doi.org/10.1111/spc3.12431 
Hehman, E., Sutherland, C. A. M., Flake, J. K., \& Slepian, M. L. (2017). The unique contributions of perceiver and target characteristics in person perception. Journal of Personality and Social Psychology, 113(4), 513-529. https://doi.org/10.1037/pspa0000090

Holzleitner, I. J., Lee, A. J., Hahn, A. C., Kandrik, M., Bovet, J., Renoult, J. P., Simmons, D., Garrod, O., DeBruine, L. M., \& Jones, B. C. (2019). Comparing theory-driven and datadriven attractiveness models using images of real women's faces. Journal of Experimental Psychology: Human Perception and Performance, 45(12), 1589-1595. https://doi.org/10.1037/xhp0000685

Hönekopp, J. (2006). Once more: Is beauty in the eye of the beholder? Relative contributions of private and shared taste to judgments of facial attractiveness. Journal of Experimental Psychology: Human Perception and Performance, 32(2), 199-209. https://doi.org/10.1037/0096-1523.32.2.199

Jones, A. L., \& Jaeger, B. (2019). Biological bases of beauty revisited: The effect of symmetry, averageness, and sexual dimorphism on female facial attractiveness. Symmetry, 11(2), 279. https://doi.org/10.3390/sym11020279

Jones, B. C., DeBruine, L. M., Flake, J. K., Liuzza, M. T., Antfolk, J., Arinze, N. C., Ndukaihe, I. L. G., Bloxsom, N. G., Lewis, S. C., Foroni, F., Willis, M. L., Cubillas, C. P., Vadillo, M. A., Turiegano, E., Gilead, M., Simchon, A., Saribay, S. A., Owsley, N. C., Jang, C., ... Coles, N. A. (2021). To which world regions does the valence-dominance model of social perception apply? Nature Human Behaviour, 5(1), 159-169. https://doi.org/10.1038/s41562-020-01007-2 
Kenny, D. A., \& Albright, L. (1987). Accuracy in interpersonal perception: A social relations analysis. Psychological Bulletin, 102(3), 390-402.

Kenny, D. A., Kashy, D. A., \& Cook, W. L. (2006). Dyadic data analysis. Guilford Press.

Kunda, Z., \& Thagard, P. (1996). Forming impressions from stereotypes, traits, and behaviors: A parallel-constraint-satisfaction theory. Psychological Review, 103(2), 284-308.

Ma, D. S., Correll, J., \& Wittenbrink, B. (2015). The Chicago face database: A free stimulus set of faces and norming data. Behavior Research Methods, 47(4), 1122-1135. https://doi.org/10.3758/s13428-014-0532-5

McCarty, M. K., \& Kelly, J. R. (2015). Perceptions of Dating Behavior: The Role of Ambivalent Sexism. Sex Roles, 72(5-6), 237-251. https://doi.org/10.1007/s11199-015-0460-6

McGraw, K. O., \& Wong, S. P. (1996). Forming inferences about some intraclass correlation coefficients. Psychological Methods, 1(1), 30-46. https://doi.org/10.1037/1082989X.1.1.30

Moshontz, H., Campbell, L., Ebersole, C. R., IJzerman, H., Urry, H. L., Forscher, P. S., Grahe, J. E., McCarthy, R. J., Musser, E. D., Antfolk, J., Castille, C. M., Evans, T. R., Fiedler, S., Flake, J. K., Forero, D. A., Janssen, S. M. J., Keene, J. R., Protzko, J., Aczel, B., ... Chartier, C. R. (2018). The Psychological Science Accelerator: Advancing psychology through a distributed collaborative network. Advances in Methods and Practices in Psychological Science, 1(4), 501-515. https://doi.org/10.1177/2515245918797607

Oosterhof, N. N., \& Todorov, A. (2008). The functional basis of face evaluation. Proceedings of the National Academy of Sciences, 105(32), 11087-11092.

Raudenbush, S. W., \& Bryk, A. S. (2002). Hierarchical linear models: Applications and data analysis methods (Vol. 1). Sage. 
Rentfrow, P. J., Gosling, S. D., \& Potter, J. (2008). A theory of the emergence, persistence, and expression of geographic variation in psychological characteristics. Perspectives on Psychological Science, 3(5), 339-369. https://doi.org/10.1111/j.1745-6924.2008.00084.x

Saribay, S. A., Biten, A. F., Meral, E. O., Aldan, P., Třebický, V., \& Kleisner, K. (2018). The Bogazici face database: Standardized photographs of Turkish faces with supporting materials. PLOS ONE, 13(2), e0192018. https://doi.org/10.1371/journal.pone.0192018

Shrout, P. E., \& Fleiss, J. L. (1979). Intraclass correlations: Uses in assessing rater reliability. Psychological Bulletin, 86(2), 420-428.

Stolier, R. M., Hehman, E., \& Freeman, J. B. (2020). Trait knowledge forms a common structure across social cognition. Nature Human Behaviour, 4(4), 361-371. https://doi.org/10.1038/s41562-019-0800-6

Stolier, R. M., Hehman, E., Keller, M. D., Walker, M., \& Freeman, J. B. (2018). The conceptual structure of face impressions. Proceedings of the National Academy of Sciences, 115(37), 9210-9215. https://doi.org/10.1073/pnas.1807222115

Sutherland, C. A. M., Burton, N. S., Wilmer, J. B., Blokland, G. A. M., Germine, L., Palermo, R., Collova, J. R., \& Rhodes, G. (2020). Individual differences in trust evaluations are shaped mostly by environments, not genes. Proceedings of the National Academy of Sciences. https://doi.org/10.1073/pnas.1920131117

Sutherland, C. A. M., Liu, X., Zhang, L., Chu, Y., Oldmeadow, J. A., \& Young, A. W. (2018). Facial first impressions across culture: Data-driven modeling of Chinese and British perceivers' unconstrained facial impressions. Personality and Social Psychology Bulletin, 44(4), 521-537. https://doi.org/10.1177/0146167217744194 
Thornhill, R., \& Gangestad, S. W. (1999). Facial attractiveness. Trends in Cognitive Sciences, 3(12), 452-460. https://doi.org/10.1016/S1364-6613(99)01403-5

Todorov, A., Olivola, C. Y., Dotsch, R., \& Mende-Siedlecki, P. (2015). Social attributions from faces: Determinants, consequences, accuracy, and functional significance. Annual Review of Psychology, 66(1), 519-545. https://doi.org/10.1146/annurev-psych-113011-143831

Vernon, R. J. W., Sutherland, C. A. M., Young, A. W., \& Hartley, T. (2014). Modeling first impressions from highly variable facial images. Proceedings of the National Academy of Sciences, 111(32), E3353-E3361. https://doi.org/10.1073/pnas.1409860111

Wang, H., Han, C., Hahn, A. C., Fasolt, V., Morrison, D. K., Holzleitner, I. J., DeBruine, L. M., \& Jones, B. C. (2019). A data-driven study of Chinese participants' social judgments of Chinese faces. PLOS ONE, 14(1), e0210315.

https://doi.org/10.1371/journal.pone.0210315

Xie, S. Y., Flake, J. K., \& Hehman, E. (2019). Perceiver and target characteristics contribute to impression formation differently across race and gender. Journal of Personality and Social Psychology, 117(2), 364-385.

Zebrowitz, L. A., Fellous, J.-M., Mignault, A., \& Andreoletti, C. (2003). Trait impressions as overgeneralized responses to adaptively significant facial qualities: Evidence from connectionist modeling. Personality and Social Psychology Review: An Official Journal of the Society for Personality and Social Psychology, Inc, 7(3), 194-215. https://doi.org/10.1207/S15327957PSPR0703_01

Zebrowitz, L. A., Wang, R., Bronstad, P. M., Eisenberg, D., Undurraga, E., Reyes-García, V., \& Godoy, R. (2012). First impressions from faces among U.S. and culturally isolated 
Tsimane' people in the Bolivian rainforest. Journal of Cross-Cultural Psychology, 43(1), 119-134. https://doi.org/10.1177/0022022111411386

Zickfeld, J., van de Ven, N., Pich, O., Schubert, T. W., Berkessel, J., Pizarro Carrasco, J. J., . ., \& Orjiakor, C. (2020). Tears trigger the intention to offer social support: A systematic investigation of the interpersonal effects of emotional crying across 41 countries. https://psyarxiv.com/p7s5v/ 